\title{
Automatic optimized 3D path planner for steerable catheters with heuristic search and uncertainty tolerance
}

\author{
Alberto Favaro $^{a}$, Leonardo Cerri ${ }^{a}$, Stefano Galvan ${ }^{b}$, Ferdinando Rodriguez y Baena $^{b}$ and Elena De Momi ${ }^{a}$
}

\begin{abstract}
In this paper, an automatic planner for minimally invasive neurosurgery is presented. The solution can provide the surgeon with the best path to connect a user-defined entry point with a target in accordance with specific optimality criteria guaranteeing the clearance from obstacles which can be found along the insertion pathway. The method is integrated onto the EDEN2020* programmable bevel-tip needle, a multi-segment steerable probe intended to be used to perform drug delivery for glioblastomas treatment. A sample-based heuristic search inspired to the BIT* algorithm is used to define the optimal solution in terms of path length, followed by a smoothing phase required to meet the kinematic constraint of the catheter. To account for inaccuracies in catheter modeling, which could determine unexpected control errors over the insertion procedure, an uncertainty margin is defined so that to include a further level of safety for the planning algorithm. The feasibility of the proposed solution was demonstrated by testing the method in simulated neurosurgical scenarios with different degree of obstacles occupancy and against other sample-based algorithms present in literature: RRT, RRT* and an enhanced version of the RRT-Connect.
\end{abstract}

\section{INTRODUCTION}

In the recent years, minimally invasive surgery (MIS) has been taking hold in hospital practice because of the unquestionable advantages for the patient. In the field of neurosurgery, common MIS include keyhole procedures such as diagnostic biopsy, Deep Brain Stimulation, Stereoelectroencephalography and drug delivery [1]. These procedures are generally performed by means of rigid linear tools which limit the usage cases to only situations where a straight line trajectories is viable.

In this context, steerable catheters can come in help as tools able to overcome limitations of the rigid embodiments, especially in scenarios where obstacles-avoidance capabilities are crucial to avoid any damage to relevant anatomical regions. In neurosurgery, such obstacles are generally represented by blood vessels, lateral ventricles, midbrain and cerebellum [2]. Many prototypes of steerable catheters have been developed for application in different surgical specialties: concentric tubes composed by multiple pre-bent stylet [3], [4], dutycycle bevel tip solutions [5-7], a tendon-actuated tip implementation [8] and a multi-segment programmable bevel-tip needle (PBN) [9]. The latter represents the case of interest

\footnotetext{
*This project has received founding from the European Unions Horizon 2020 research and innovation program under grant agreement No 688279.

${ }^{a}$ Department of Electronics, Information and Bioengineering, Politecnico di Milano, Milan, Italy alberto.favaro@polimi.it elena.demomi@polimi.it

${ }^{b}$ Mechatronics In Medicine Laboratory, Mechanical Engineering Department, Imperial College London, London SW7 2AZ, U.K. (e-mail: f.rodriguez@imperial.ac.uk)
}

of this work: it consists in the bio-inspired EDEN2020* catheter [10], a steerable needle composed by four axially interlocked segments whose degree of steering is a function of the offset between the needle segments at the catheter tip. EDEN2020 catheter finds a direct application scenario in drug delivery in glioblastomas treatments, but can also see possible implementations in tumor treatment, brachytherapy and diagnostic biopsy of cancerous tissue.

An intelligent planner can result useful in assisting the surgeon to define the best surgical trajectory to perform, giving the possibility to automatically estimate a viable pathway in accordance with kinematic constraints of the catheter and refining the solution in accordance with optimality criteria as the total path length and the distance from the safety-critical obstacles.

The present work aims to describe an automatic 3D path planning solution for robot-assisted neurosurgery. This algorithm is designed to meet PBN's kinematic constraints and non-holonomicity and to guarantee a high reliable level of obstacles-avoidance capability, crucial for the intended neurosurgical application, through the definition of a proper uncertainty margin designed to account for inaccuracies in the catheter modeling which can result in possible obstacles collisions.

The proposed automatic planner exploits the asymptoticallyoptimum planning solution described in [11] for estimating an obstacle-free raw path to solve a single-query planning task (i.e. to connect a start point to a goal) and performs a path optimization according to a cost function in order to maximize the obstacle clearance, reducing the total path length and meeting PBN's curvature limit. The proposed solution comes as a 3D Slicer(c) (www.slicer.org) module. The paper is structured as follows. In Section II an overview of the current approaches to path planning is given, including solutions specifically intended for MIS applications. Section III provides a description of the presented approach: the planning problem, the path smoothing, the implementation of the uncertainty margin and the definition of a cost function. Results from simulations are presented in Section IV; discussion and conclusions can be found respectively in in Section V and Section VI.

\section{RELATED WORKS}

In the context of path planning, a variety of approaches has been proposed in literature. Duindam et al. in [12] describe an inverse kinematics solution to the problem of estimating a catheter pathway, the method was tested in a simplified environment with obstacles represented by geometrically-shaped 
object showing limited obstacles-avoidance capabilities. In [13], an algorithm for MIS trajectories based on a probability map is presented, but the solution has not been tested in presence of obstacles.

Potential field methods, originally introduced in [14], are based on the computation of a field that increases getting closer to the obstacles, which has the disadvantage of determining local minima. To address this problem, Li et al. [15] suggested an application for brachytherapy procedures with obstacle avoidance capability based on an artificial potential field where a conjugate gradient algorithm is used. Clearance from anatomical structures is achieved, but the path can not be optimized for optimality criteria as the total trajectory length.

Further solutions presented in literature can be divided in two main categories: graph-based and sampling-based methods.

\section{A. Graph-based methods}

Dijkstra algorithm [16] and $A^{*}$ [17] are two typical graph-search methods based onto the discrete approximation of the planning problem. They are "resolution-complete" algorithms, as they can determine in finite time weather a solution exists, and "resolution-optimal" since they can estimate the best path given the specific resolution of the approximation.

An incremental $\mathrm{A}^{*}$ solution for 2D applications was proposed Likhachev et al. [18] which reuses previous information and drives the path towards the optimality.

The relative simpleness of these methodologies conflicts with the high computation time necessary to solve the optimal planning problem in high dimensional cases, as the discretization of the environment becomes finer. For this reason, they are not suitable for a neurosurgical application, where the search for optimality requires to densely sample the working domain.

Discretization of the working domain in subspaces is the base of Adaptive Fractal Tree [19], which exploits fractal theory and parallelization to process them separately and builds a tree composed by arcs with bounded curvature. It focuses the research toward the goal guaranteeing a computational time compatible with real time, computer-assisted MIS, but it needs a performing GPU to cope with the domain discretization.

\section{B. Sampling-based methods}

In presence of kinematic constraints, as in the case of steerable MIS probes, the current trend in path planning is represented by sampling-based methods. Based on the random sampling of the working space, they avoid the discretization typical of graph-based solutions. Rapidlyexploring Random Trees (RRT) and RRT-Connect [20] are sampling-based solution able to scale more effectively with high dimensional query problems. Their enhanced versions, RRT* [21] and bidirectional-RRT [22], are probabilistically complete as they have a probability which tends to one to find a solution, if it exists, as the number on samples goes to infinity. Moreover, they are also asymptotically optimal as they can refine the initially-estimated raw path when new points are sampled, providing the shortest solution to the query problem at the limit. A combination of RRT and a reachability-guided sampling heuristic (RG-RRT) is used in the work of Patil et al. [23] to compute motion plans for steerable needles in complex 3D environments by constructing the tree via a sequential connection of arcs with bounded curvature. Fast computation allows these solutions to be used in real time applications, but efficacy test have been carried out only in a simplified space with few spherical and cylindrical objects. A neurosurgical implementation of RG-RRT is the one proposed by Caborni et al. in [24], but the solution estimates trajectories only in $2 \mathrm{D}$ space.

Gammell at al. [11] proposed the Batch Informed Tree (BIT*). The algorithm balances the benefits of a graphsearch approach as it originally creates a graph solved through an incremental variation of the $A^{*}$, and advantages of sampling-based algorithms since it asymptotically finds the optimal query-problem solution in terms of path length by increasingly sampling the working domain. As soon as a first raw path is found, BIT* confines the research within an ellipsoidal region whose size depends on the cost of the current solution so that the research for a shorter path occurs only within a subspace of the working domain.

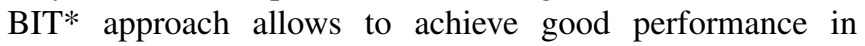
terms of computational time with respect to other standard sampling-based algorithms but its feasibility has never been assessed in MIS automatic planning, where multiple other parameters have to be considered in addition to the path length.

In this paper, a novel 3D MIS path planner for neurosurgical application is presented. It exploits the search approach implemented in $\mathrm{BIT}^{*}$, adapting the solution to limits determined by PBN and optimizing the path not only for the length but also for kinematic constraints and obstacles clearance capability required by the intended application.

\section{METHODS}

\section{A. The workflow}

At first, the single-query problem is solved through an implementation of the BIT* approach, which generates a set of feasible paths (Section III-B, III-C, Step 2 of Fig.1).

In order to guarantee a $\mathrm{C}^{2}$ continuity, the estimated path undergoes a cardinal spline interpolation and an uncertainty margin is built around them to account for catheter model inaccuracies (Section III-D, Step 3 of Fig.1).

Limits to the path curvature related to kinematic constraints of the catheter are addressed in Section III-E, where paths smoothing is described (Step 4 of Fig.1). This step is useful also for maintaining the path the straighter as possible, as large degree of steering is shown to be correlated to greater trajectory tracking errors [25]. Smoothing stops when curvature reduction drives the path too close to an obstacles or when it does not produce further relevant changes in the curvature.

The last step consists in ranking the obtained paths. At this stage, the estimated solutions meet both kinematic 


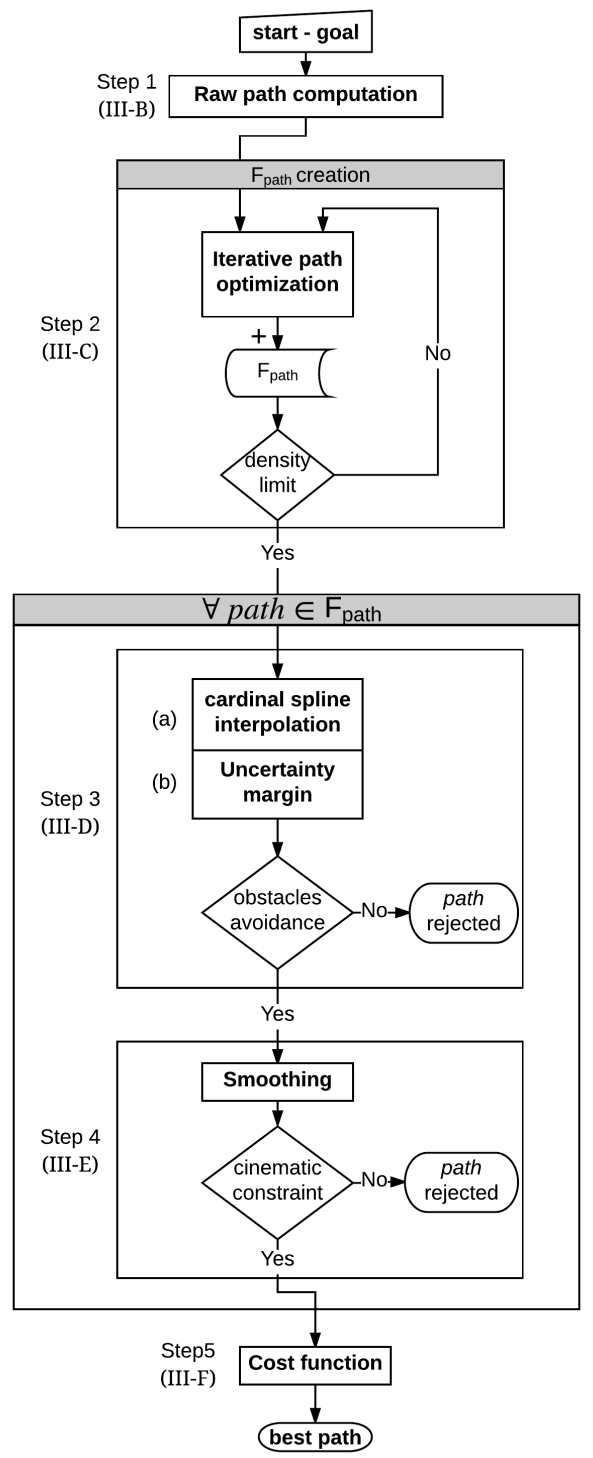

Fig. 1: Schematic representation of the workflow for the presented solution.

constraints and obstacles avoidance. In Section III-F the cost function implemented to find the best solution to the query problem is described (Step 5, Fig.1).

\section{B. Path search}

At first, a distance map (dist $\left.{ }_{\text {map }}\right)$ is estimated on the 3D working domain. The map labels each voxel with the Euclidean distance to the nearest obstacle and it is interrogated at each iteration to ensure that new segments added to the path are safe in terms of obstacles avoidance.

An initial ellipsoid $\mathcal{H}$ is built similarly to [11] as:

$$
X=\left\{\mathbf{x} \in X:\left\|\mathbf{x}_{\text {start }}-\mathbf{x}\right\|_{2}+\left\|\mathbf{x}-\mathbf{x}_{\text {goal }}\right\|_{2} \leqslant c_{\text {best }}\right\}
$$

where the focal length is set as the Euclidean distance between the start and goal points, and a preset value is given to the minor axis.

A uniform random distribution of samples $\mathcal{U}$ are taken within
$X_{\text {free }}^{\mathcal{H}}$, defined as:

$$
X_{\text {free }}^{\mathcal{H}}=\left\{\mathbf{p} \in\left(X_{\mathcal{H}} \cap X_{\text {free }}\right)\right\}
$$

where $X_{\mathcal{H}}$ is the subspace of the working domain contained in $\mathcal{H}$ and $X_{\text {free }}$ the subspace represented by the obstaclesfree voxels.

For each randomly-sampled point $\mathbf{p}_{\text {new }}$ in $X_{\text {free }}^{\mathcal{H}}$, a set of neighbors $P_{n}$ is defined as:

$$
P_{n}=\left\{\mathbf{p} \in G:\left\|\mathbf{p}_{\text {new }}-\mathbf{p}\right\|<r\right\}
$$

where $G$ represents the connected graph built within the ellipsoid through an RRT $^{*}$ approach and $r$ is a fixed radius of a sphere; $\mathbf{p}_{\text {new }}$ is connected to the point of $G$ included in the set $P_{n}$ which minimize the total length from start to $\mathbf{p}_{\text {new }}$ while maintaining the new connection at a distance $d>D_{\text {safe }}$ from obstacles. When all the points of $\mathcal{U}$ have been inspected and, if safely attachable, added to $G$, the algorithm tries to connect goal to $G$. If this step goes though, a first raw solution to the query problem is found and the algorithm proceeds to the optimization phase (Section III-C). Otherwise, a larger ellipsoid $\mathcal{H}_{\text {new }}$ is defined, widening the subset of the working domain where the query-problem solution can lie and a new set of points are randomly sampled within $\mathcal{H}_{\text {new }}$ and added to $\mathcal{U}$ maintaining an uniformed distribution. $G$ is thus populated with new nodes and the search for the query solution repeats until a path is found or the number of iterations reaches a limit, meaning that the algorithm could not solve the planning problem (Step 1, Fig.1).

\section{Path optimization}

As the first feasible solution is found, path, it is stored in a set of feasible paths $\left(F_{\text {paths }}\right)$ and its length, $l_{\text {path }}$, is computed. A new ellipsoid $\mathcal{H}_{\text {new }}$ is defined by setting the major axis equal to $l_{\text {path }}$ and $\mathcal{U}$ is populated with a new set $\mathcal{U}_{\text {new }}$ of uniformly distributed samples taken within $\mathcal{H}_{\text {new }}$ so that $\mathcal{U} \leftarrow \mathcal{U}_{\text {new }}$. If a shorter path exists, it can only lies within $\mathcal{H}_{\text {new }}$, as demonstrated in [26].

Path optimization (Step 2, Fig.1) is presented in Algorithm 1 and herein discussed. An illustration of the method is shown in Fig.2.

Each new point $\mathbf{p}_{\text {new }} \in \mathcal{U}$ which has never been examined is add to $G$ following an RRT* approach similarly to IIIB (Fig.2b, Lines 2-4). At Line 5, the subset of points $G_{n}$ of path in proximity of $\mathbf{p}_{\text {new }}$ and lying within a sphere of radius $r$ is defined. For each node $\mathbf{g}_{n} \in G_{n}$, the segment from $\mathbf{p}_{n e w}$ to $\mathbf{g}_{n}$ is checked for its clearance from obstacles (Lines 6-7). If $\mathbf{g}_{n}$ is safe, the length $d_{\text {goal }}$ of the connection between $p_{n e w}$ and goal over the graph $G$ passing though $\mathbf{g}_{n}$ is determined (Line 8 ). In case $d_{\text {goal }}$ represents the shortest pathway from $p_{n e w}$ to goal, $\mathbf{g}_{\mathbf{n}}$ is stored in $\mathbf{g}_{\text {best }}$ (Fig.2c, Lines 9-11). When all the points in $G_{n}$ has been checked, $p_{\text {new }}$ is connected to $G$ though $\mathbf{g}_{\text {best }} . G$ is then updated (Fig.2d, Line 12) and in case a new shorter solution to the query problem is found, this is stored in path (Lines 13-14), resulting in focusing research to smaller ellipsoidal region, and the new solution is pushed in the set of feasible path 


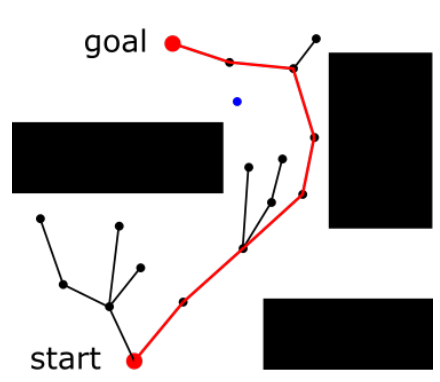

(a)

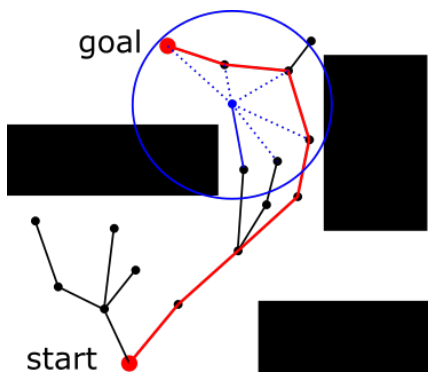

(b)

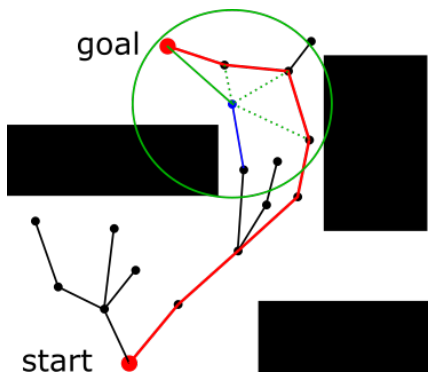

(c)

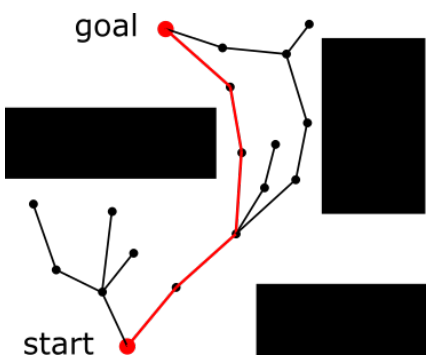

(d)

Fig. 2: Illustration of the optimization algorithm in a simple $2 \mathrm{D}$ environment. The start and goal points are shown in red dots, the red path represents the current best solution and the blue point the new sample to be added to the graph (Fig.2a). Fig.2b shows the connection of the new sample to the existing tree by choosing among the neighbors the one that minimizes the total length from start. Fig.2c depicts the research for a better solution: the new sample is linked to the neighbor which minimizes the total length to the goal. The updated best path is shown in Fig.2d.

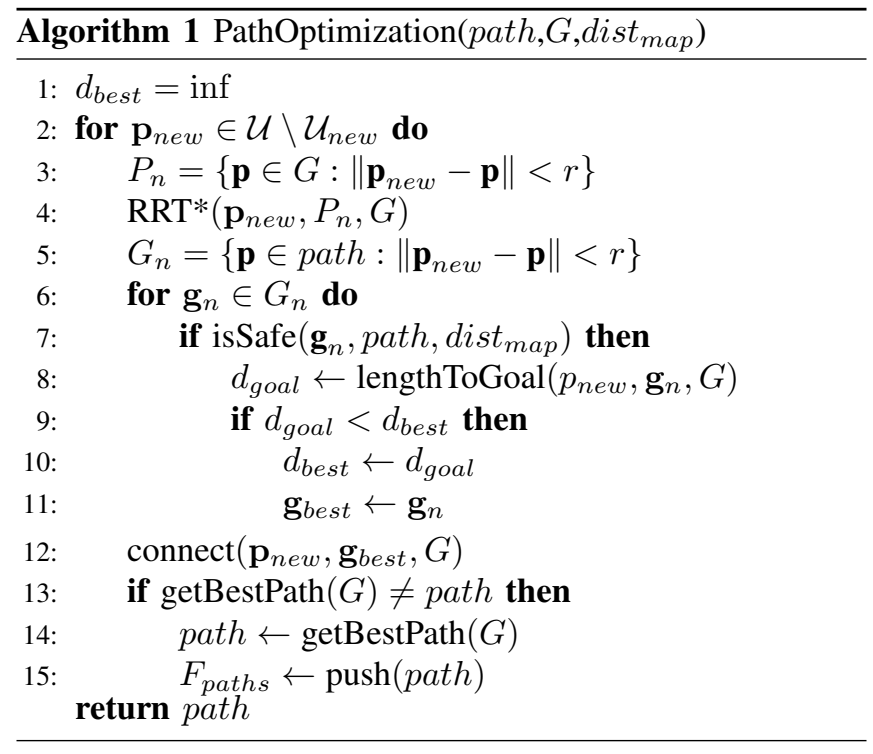

$F_{\text {paths }}$.

Optimization repeats until the density of samples in $\mathcal{U}$ has reached a predefined threshold.

As points in $\mathcal{U}$ are uniformly sampled within subspaces of the working domain and a RRT* strategy is used to populate the graph $G$, Algorithm 1 herein proposed will converge asymptotically to the shortest query problem solution as the number of samples in $\mathcal{U}$ goes to infinity [21].

\section{Path interpolation and uncertainty margin}

The result of Section III-C consists in a set $F_{\text {path }}$ of feasible paths able to solve the planning problem. Each of them represents a piecewise-linear trajectory. To achieve the $\mathrm{C}^{2}$ continuity required by the catheter, a cardinal spline interpolation is carried out on paths in $F_{\text {path }}$ using path nodes as control points (Step 3a, Fig.1).

To account for inaccuracies in catheter modeling and add a further level of safety with respect to the strong obstacles clearance required by the presented MIS application, an uncertainty margin is built (Step 3b, Fig.1) around each interpolated trajectory in $F_{\text {path }}$ (Fig.3). Similarly to [27], where a non-holonomic steerable needle is used as test case for path planning simulations under uncertainties, here a zero-mean Gaussian distribution is considered to model the motion error $\mathbf{m}$ with a variance $\mathbf{M}$ equal to $0.001 \mathrm{~mm}^{2}$.

$$
\mathbf{m} \sim \mathcal{N}(\mathbf{0}, \mathbf{M})
$$

A confidence bound $c b$ of one standard deviation is considered, a catheter insertion speed speed of $3 \mathrm{~mm} / \mathrm{s}$ and it is supposed that the robot position is periodically checked with a sampling time $\boldsymbol{\Delta}=0.5 \mathrm{~s}$. This results in an increase in the confidence bounds of one $c b$ every $1.5 \mathrm{~mm}$ of path length. A continuous representation of the uncertainty margin is obtained by linearly interpolating $c b$ from one time step to the other resulting in a cone whose radius enlarges in approaching the goal point.

All paths in $F_{\text {path }}$ are then checked to verify whether they are still safe after the implementation of the uncertainty margin. Solutions which result unfeasible at this stage are discarded.

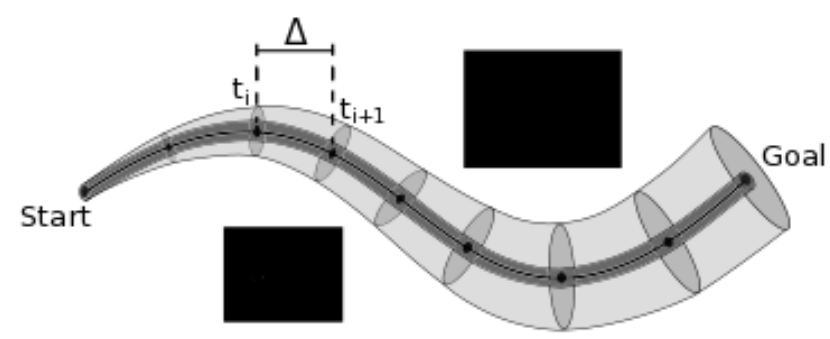

Fig. 3: Illustration of the uncertainty margin. The solid black line represents the estimated trajectory after the interpolation step while the constant-radius tube symbolizes the actual catheter size.

\section{E. Path smoothing}

To meet the limit of the catheter curvature, a smoothing phase is then accomplished (Step 4, Fig.1). The smoothing solution is presented in Algorithm 2 and described hereinafter. Fig. 4 illustrates the idea behind the method.

The smoothing algorithm receives one by one the paths of $F_{\text {path }}$ to correct and the distance map. The curvature $K$ is computed over the whole path under examination and points that do not satisfy kinematic constraints $\left(\mathrm{s} \in\right.$ path $: K_{\mathbf{s}}>$ 


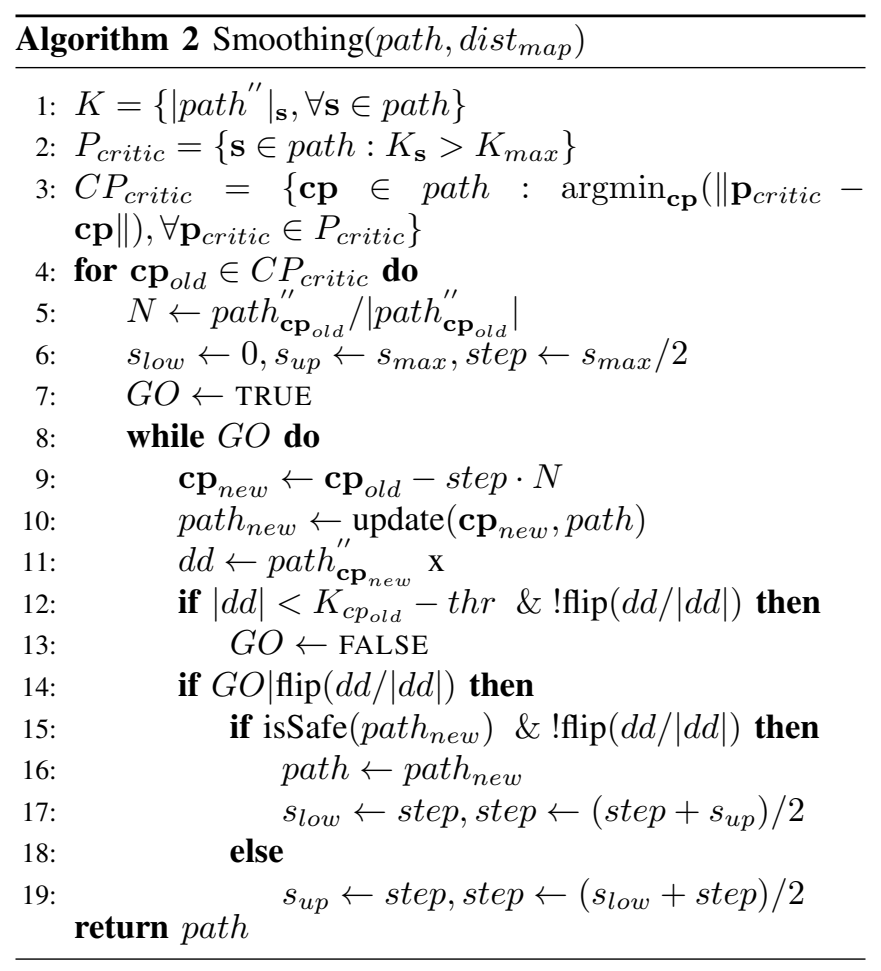

$\left.K_{\max }\right)$ are linked to their closest control point cp so that a set $C P_{\text {critic }}$ of control points that required revision is defined (Lines 1-3).

For each cp $\in C P_{\text {critic }}$ (Line 4) the principal normal vector $N$ to the curve is determined as the direction along which cp has to be moved in order to decrease $K_{\mathbf{c p}}$ (Line 5): exploiting a bisection approach, the method will try to move cp as much as possible along $N$ (up to a preset limit $s_{\max }$ ) maintaining the required clearance from obstacles. At Lines 6-7, parameters linked to cp displacement are set, as well as a flag variable used to terminate the iterations.

Smoothing of path is accomplished within Line 8 and 19. The process starts by moving $\mathbf{c p}$ to $\mathbf{c p}_{n e w}$ along $N$ by a certain amount of space defined by step (Line 9). A new cardinal spline interpolation and the related uncertainty margin are computed, identified as path new $_{\text {(Line 10) and }}$ the new second derivative $d d$ in $\mathbf{c p}_{\text {new }}$ is calculated (Line 11).

If the stop condition is reached, i.e. the curvature reduction results to be too small (Line 12), the correction of the current cp stops. More than the check of the curvature variation, in Line 12 the function flip verifies whether $\mathbf{c p}_{\text {new }}$ has been moved so far that the curvature has changed its sign (in Fig. 4 this condition happens when the curve is flipped downside), in this case the step has to be reduced and the algorithm will move directly to Line 19.

In case the uncertainty margin related to $p^{2} h_{n e w}$ maintains a safety distance from obstacles after cp adjustment, path is updated and step is resized in order to move cp further in the same direction (Lines 15-17).

Differently, in case of a collision with an obstacle or a change in the curvature sign, the upper limit $s_{u p}$ is reduced

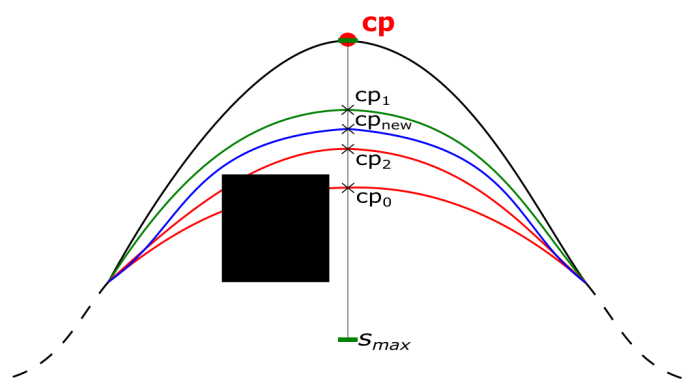

Fig. 4: Description of the curvature correction algorithm: the control point $\mathbf{c p}$ is moved to the new position $\mathbf{c p}_{0}$ as the midpoint between $\mathbf{c p}$ and $s_{\max }$. The new path computed through $\mathbf{c p}_{0}$, in red line, results not safe and a the new position $\mathbf{c p}_{1}$ is searched by decreasing the step size at the midpoint between $\mathbf{c p}$ and $\mathbf{c p}_{0}$. The resulting path is now feasible (green line), and a smoother solution is searched at the midpoint between $\mathbf{c p}_{0}$ and $\mathbf{c p}_{1}$. The stop condition is reached when $\mathbf{c p}$ is moved to $\mathbf{c p}_{\text {best }}$ and path is accordingly modified (blue line).

and step is shortened as the midpoint between $s_{l o w}$ and its current value.

The smoothing of path repeats until the stop condition is reached and all the critical points $\mathbf{c p} \in C P_{\text {critic }}$ are corrected.

\section{F. Cost function}

Section III-E updates $F_{\text {paths }}$ with smoothed trajectories able to solve the query problem through feasible pathways which guarantee obstacles clearance and the meet of curvature limits.

In order to rank these solutions, a proper cost function (Step 5, Fig.1) is introduced so that the optimum path in $F_{\text {paths }}$ will be the one that minimizes $F_{\text {cost }}$, defined as:

$$
F_{\text {cost }}=\alpha \cdot \frac{l}{l_{m}}-\beta \cdot \frac{d_{m}}{d_{M}}-\gamma \cdot \frac{\bar{d}}{d_{M}}+\delta \cdot k_{M}
$$

where $l$ is the total path length, $l_{m}$ the Euclidean distance between start and goal,,$d_{m}$ and $\bar{d}$ the minimum and mean distances from obstacles over the whole path length, $d_{M}$ the maximum value stored in dist $_{\text {map }}$ and $k_{M}$ the maximum curvature reached along the path, while $\alpha, \beta, \gamma, \delta$ are the parameters weights respectively equal to $1,0.8,0.2$, $1 / \mathrm{mm}^{-1}$.

\section{Results}

The feasibility of the proposed method was assessed via simulations. In light of this, two Magnetic Resonance Imaging (MRI) acquisition protocols have been employed to collect the data required for defining a typical neurosurgical environment. A T1-weighted volumetric acquisition (Philip Ingenia CX 3T, TR/TE (ms): 12/5.8, data matrix: $320 \times 299$, FOV $(\mathrm{mm}): 256 \times 240$, in-plane resolution $(\mathrm{mm}): 0.80 \times 0.80$, thickness (mm): 0.80, number of sections: 236) was used for the definition of the brain volume, whilst a 3D highresolution time-of-flight (TOF) acquisition (Philip Ingenia CX 3T, TR/TE (ms): 23/3.5, acquisition plane: axial, data matrix: $500 \times 399$, FOV $(\mathrm{mm}): 200 \times 200$, in-plane resolution $(\mathrm{mm}): 0.40 \times 0.50$, thickness $(\mathrm{mm}): 0.90$, number 

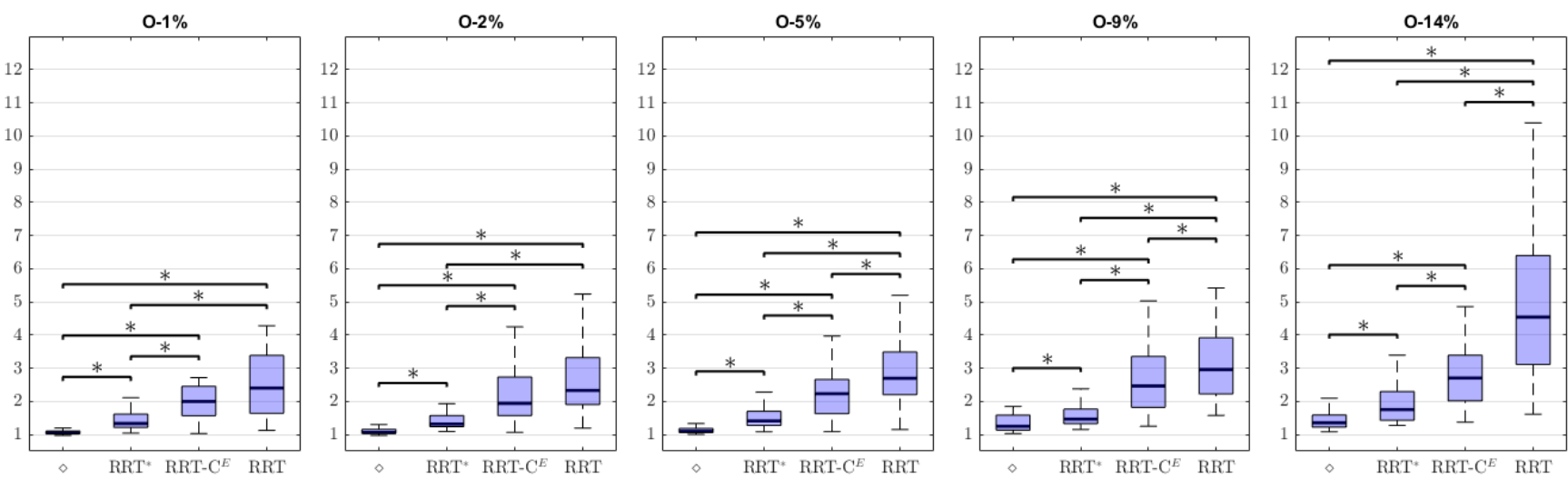

Fig. 5: The presented solution $(\diamond)$ is tested against three different planning algorithms: RRT, RRT*, RRT-Connect ${ }^{\mathrm{E}}$ in five 3D scenarios with different percentages of space occupancy: $1 \%, 2 \%, 5 \%, 9 \%$ and $14 \%$. Algorithms is asked to solve a total of 50 queries for each scenario and each time the first raw path is evaluated. The box-plots resume the obtained results in term of cost function 4), statistical significance between different algorithms is also reported.

of sections: 210) allowed the identification of the cerebral arteries, which represent the obstacles for the planning algorithm. Brain volume and arterial vessels segmentations were performed manually though 3D Slicer. Data acquisitions from one healthy subject were performed at the Center of Excellence for High Field Magnetic Resonance (CERMAC), Ospedale San Raffaele, Milan, Italy under ethical approval n.80/INT/2016 and patient gave written informed consent. The experimental setup consists in testing the algorithm on a set of 50 single queries (the combinations of start and goal), placed within the working scenario at different MISlike locations. The arterial vessels tree, which represents the original working scenario, has been incrementally magnified so that to dispose of a total of 5 different working environment, where the obstacles occupancy ranges from $1 \%$ (i.e the original angiography, case $\mathrm{O}-1 \%$ ) to $14 \%$ (larger angiography dilation, case $\mathrm{O}-14 \%$ ). As testing environment, 3DSlicer(C) has been used.

Tests were run on iMac (OS-X 10.11.6, 3.1GHz Intel Core i7, 8GB of RAM). The initial minor axis of the starting searching ellipsoid defined in Sec.III-B was set equal to $10 \mathrm{~mm}$ (1). The initial number of samples in $\mathcal{U}$ was set to 5 , the radius $r$ to $40 \mathrm{~mm} 3, D_{\text {safe }}$ to $0.25 \mathrm{~mm}$ and a threshold for the density of points in $\mathcal{U}$ are to 0.01 sample $/ \mathrm{mm}^{3}$. The smoothing algorithm in III-E uses a $s_{\max }$ equal to $10 \mathrm{~mm}$, the maximum curvature $K_{\max }$ was set to $0.014 \mathrm{~mm}^{-} 1$ as the reported in [19], and the threshold for sensible curvature variation thr to $0,001 \mathrm{~mm}^{-1}$.

\section{A. Comparison with solutions from literature}

A first test has been carried out to compare the results in terms of cost function obtained by the presented solution against existing sample-based planning algorithms: RRT,

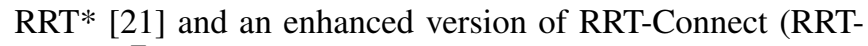
Connect $^{\mathrm{E}}$ ). The latter consists in a standard RRT-Connect solution [20], consisting in two graphs rooted at the start and goal point spanning the working domain to connect to each other, to which a $r$-radius sphere is added to prune the graph, as in III-B.
As RRT and RRT - Connect $^{\mathrm{E}}$ are not incremental algorithms, they do not refine the first raw path even if new points are sampled in the working domain. For this reason, this test analyses only the first raw solution discovered by the four algorithms to the query problems (Step 1, Fig.1) to which a cardinal interpolation is applied (Step 3a, Fig.1) in oder to let the computation of the path curvature be possible.

Fig. 5 shows the obtained results. We evaluated the performance of the four different algorithms by referring to the cost computed by the cost function. As the variable cost was not normally distributed (lilliefors test, $\mathrm{p}<0.05$ ), we ran non-parametric statistics. The Kruskal-Wallis test highlighted significant effects of the algorithm over the performance in all the different working domains $(\mathrm{H}(3,196)>98.95, \mathrm{p}<0.05)$. To evaluate differences between each pair of methods, we ran pairwise comparisons through Mann-Whitney $\mathrm{U}$ test $(\mathrm{U}(1,98)>18, \mathrm{p}<0.0125)$. As can be noticed, the heuristic search implemented in our solution (identified as $\diamond$ in Fig.5) leads to a first raw path which cost is globally smaller with respect to other algorithms for all the working scenarios.

\section{B. Test of the algorithm workflow}

Tests have been carried to verify the feasibility of the proposed method in its entirely, i.e. following all the step described in the workflow of Section III-A and depicted in Fig.1. The 50 single-queries are provided again to our

TABLE I: Success rate and computational time for the test of the algorithm workflow

\begin{tabular}{ccccccc}
\hline & \multicolumn{3}{c}{ Comput. Time } & \multicolumn{3}{c}{ Constraints compliance } \\
Case & $25^{t h}$ & median & $75^{t h}$ & Geom. & Cin. & Overall \\
\hline $\mathrm{O}-1 \%$ & $21.2 \mathrm{~s}$ & $42.2 \mathrm{~s}$ & $71.4 \mathrm{~s}$ & $90 \%$ & $14 \%$ & $12 \%$ \\
$\mathrm{O}-2 \%$ & $32.7 \mathrm{~s}$ & $45.8 \mathrm{~s}$ & $65.5 \mathrm{~s}$ & $76 \%$ & $2 \%$ & $2 \%$ \\
$\mathrm{O}-5 \%$ & $35.9 \mathrm{~s}$ & $56.6 \mathrm{~s}$ & $85.4 \mathrm{~s}$ & $76 \%$ & $0 \%$ & $0 \%$ \\
$\mathrm{O}-9 \%$ & $38.5 \mathrm{~s}$ & $66.0 \mathrm{~s}$ & $96.5 \mathrm{~s}$ & $52 \%$ & $0 \%$ & $0 \%$ \\
$\mathrm{O}-14 \% \bullet$ & $45.1 \mathrm{~s}$ & $71.9 \mathrm{~s}$ & $115.2 \mathrm{~s}$ & $38 \%$ & $0 \%$ & $0 \%$ \\
\hline
\end{tabular}


TABLE II: Path costs and cost function parameters for the test of the algorithm workflow

\begin{tabular}{|c|c|c|c|c|c|c|c|c|c|c|c|c|c|c|c|}
\hline \multirow[b]{2}{*}{ Case } & \multicolumn{3}{|c|}{ Overall Cost } & \multicolumn{3}{|c|}{ Length $[\mathrm{mm}]$} & \multicolumn{3}{|c|}{ D min $[\mathrm{mm}]$} & \multicolumn{3}{|c|}{ D mean $[\mathrm{mm}]$} & \multicolumn{3}{|c|}{$K \max [1 / \mathrm{mm}]$} \\
\hline & $25^{t h}$ & median & $75^{t h}$ & $25^{t h}$ & median & $75^{t h}$ & $25^{t h}$ & median & $75^{t h}$ & $25^{t h}$ & median & $75^{t h}$ & $25^{t h}$ & median & $75^{t h}$ \\
\hline $\mathrm{O}-1 \%$ & 0.979 & 0.980 & 0.990 & 66.5 & 74.1 & 81.8 & 0.82 & 1.12 & 1.63 & 6.47 & 7.13 & 7.65 & 0.016 & 0.026 & 0.056 \\
\hline $\mathrm{O}-2 \%$ & 1.001 & 1.001 & 1.001 & 70.1 & 77.3 & 81.1 & 1.18 & 1.34 & 1.49 & 5.45 & 6.03 & 6.71 & 0.029 & 0.041 & 0.066 \\
\hline $\mathrm{O}-5 \% \bullet$ & 1.015 & 1.038 & 1.063 & 69.4 & 77.9 & 82.5 & 1.19 & 1.43 & 1.84 & 4.81 & 5.64 & 6.73 & 0.040 & 0.068 & 0.185 \\
\hline $\mathrm{O}-9 \% \bullet$ & 1.058 & 1.083 & 1.195 & 68.2 & 76.5 & 83.5 & 1.15 & 1.33 & 1.51 & 4.89 & 5.60 & 5.99 & 0.051 & 0.111 & 0.163 \\
\hline $\mathrm{O}-14 \%^{\bullet}$ & 1.093 & 1.122 & 1.221 & 78.9 & 86.2 & 94.6 & 1.27 & 1.37 & 1.51 & 4.54 & 4.75 & 5.08 & 0.066 & 0.088 & 0.164 \\
\hline
\end{tabular}

algorithm in the 5 different working domains (from case O$1 \%$ to $\mathrm{O}-14 \%$ ).

Table I shows the results for the 5 different domains. The computational time required to perform the entire workflow is reported in the first column with its $25 \%$ quartile, median value and $75 \%$ quartile. Success rate is also presented in the second column in terms of compliance with respect to the geometric and the kinematic constraints, respectively represented by the obstacles avoidance and maximum curvature admitted by the catheter. Case $\mathrm{O}-5 \%, \mathrm{O}-9 \%$ and $\mathrm{O}-$ $14 \%$ consist in scenarios where the space occupancy of the obstacles precludes the possibility to solve the planning problem, these cases have been identified with $\left({ }^{\bullet}\right)$. Table I reports the simulations results in terms of cost function and related parameters. In cases $(\bullet)$, where no solution has been found that fulfills the curvature limit, the cost function and related parameters are computed only considering the paths that meet the geometric constraint of obstacles avoidance.

\section{DISCUSSION}

When compared with other sampling-based algorithms, simulations demonstrated that the proposed method outperformed other approaches in all the different domains, estimating the best raw path according to the cost function. This outcome can be explained by the heuristic implemented in our solution that, differently from RRT, RRT* and RRTConnected $^{\mathrm{E}}$, focuses the research to a subspace defined by the ellipsoid having start and goal points as focal length. When the aim was to test the entire workflow, simulations demonstrated the feasibility of the method in a standard neurosurgery working scenario (case O-1\%), where the $12 \%$ of the queries resulted connectible through feasible paths. The number decreases to only one query in case $\mathrm{O}-2 \%$, where the obstacles size were duplicated. Despite the acceptable results in terms of geometric constraints compliance, our method was not able to find a feasible solution in scenarios with denser obstacles occupancy (case 0-5\%, case O-9\%, case O-14\%) and this is due to PBN's strict curvature limit. In these tests, computational time ranges between few dozens of seconds up to some minutes depending on the working scenarios. This can be explained by the number of steps composing the workflow, the computational time required for resizing the ellipsoid in Section III-B and the need to repeat steps 3 and 4 of Fig. 1 for each path in $F_{\text {path }}$.

\section{CONCLUSIONS}

The present work proposes a novel automatic planner for minimally invasive neurosurgery. The solution can provide the surgeon with feasible paths in accordance with the kinematic limits of the catheter to implant and the obstacles clearance required by the intended application. Exploiting an heuristic search based on [11], the algorithm can save time in searching for better paths by focusing only to volume where a better solution can lies, incrementally shortening the initial raw path as the samples density of the working domain becomes finer.

An uncertainty margin is implemented to increase the safety limit and thus to address possible control errors which can occur during the implantation phase due to catheter model inaccuracies.

A bespoke cost function is then used to sort the set of feasible trajectories and provide the surgeon with the optimal path. Integrated onto PBN, the method showed good results in finding a solution to the query problems despite the strict curvature limit imposed by the catheter kinematic and, when inquire only for a unoptimized solution (Step 1 if Fig. 1), it outperformed other searching algorithms presented in literature in terms of cost function. Computational time can be ameliorated by the reduction the sequence of steps composing the workflow: using an approach similar to [19], [24] arcs with bounded curvature can be used instead of straight segments to directly build both a curvilinear path and the uncertainty margin, without the need of any interpolation step.

Moreover, the search for the first raw path described in Section III-B can be accelerated by the implementation of a bidirectional approach.

In addition to modeling errors, the noise related to the catheter sensing system can be considered in the definition of the uncertainty margin which would add a further level of safety to the automatic planner.

\section{REFERENCES}

[1] N. Abolhassani, R. Patel, and M. Moallem, "Needle insertion into soft tissue: A survey," Medical engineering \& physics, vol. 29, no. 4, pp. 413-431, 2007.

[2] E. De Momi, C. Caborni, F. Cardinale, G. Casaceli, L. Castana, M. Cossu, R. Mai, F. Gozzo, S. Francione, L. Tassi, G. Lo Russo, L. Antiga, and G. Ferrigno, "Multi-trajectories automatic planner for stereoelectroencephalography (seeg)," International Journal of Computer Assisted Radiology and Surgery, vol. 9, no. 6, pp. 10871097, Nov 2014. [Online]. Available: https://doi.org/10.1007/s11548 014-1004-1

[3] P. E. Dupont, J. Lock, B. Itkowitz, and E. Butler, "Design and control of concentric-tube robots," IEEE Transactions on Robotics, vol. 26, no. 2, pp. 209-225, 2010.

[4] D. C. Rucker, B. A. Jones, and R. J. Webster, "A model for concentric tube continuum robots under applied wrenches," in Robotics and Automation (ICRA), 2010 IEEE International Conference on. IEEE, 2010, pp. 1047-1052. 
[5] J. A. Engh, D. S. Minhas, D. Kondziolka, and C. N. Riviere, "Percutaneous intracerebral navigation by duty-cycled spinning of flexible bevel-tipped needles," Neurosurgery, vol. 67, no. 4, pp. 1117-1123, 2010.

[6] V. Kallem and N. J. Cowan, "Image guidance of flexible tip-steerable needles," IEEE Transactions on Robotics, vol. 25, no. 1, pp. 191-196, 2009.

[7] K. B. Reed, A. Majewicz, V. Kallem, R. Alterovitz, K. Goldberg, N. J Cowan, and A. M. Okamura, "Robot-assisted needle steering," IEEE robotics \& automation magazine, vol. 18, no. 4, pp. 35-46, 2011.

[8] P. Qi, H. Liu, L. Seneviratne, and K. Althoefer, "Towards kinematic modeling of a multi-dof tendon driven robotic catheter," in Engineering in Medicine and Biology Society (EMBC), 2014 36th Annual International Conference of the IEEE. IEEE, 2014, pp. 3009-3012.

[9] A. Leibinger, M. J. Oldfield, and F. R. y Baena, "Minimally disruptive needle insertion: a biologically inspired solution," Interface focus, vol. 6 , no. 3 , p. $20150107,2016$.

[10] http://www.eden2020.eu.

[11] J. D. Gammell, S. S. Srinivasa, and T. D. Barfoot, "Batch informed trees (bit*): Sampling-based optimal planning via the heuristically guided search of implicit random geometric graphs," in Robotics and Automation (ICRA), 2015 IEEE International Conference on. IEEE, 2015, pp. 3067-3074.

[12] V. Duindam, J. Xu, R. Alterovitz, S. Sastry, and K. Goldberg, "Threedimensional motion planning algorithms for steerable needles using inverse kinematics," The International Journal of Robotics Research, vol. 29, no. 7, pp. 789-800, 2010.

[13] W. Park, J. S. Kim, Y. Zhou, N. J. Cowan, A. M. Okamura, and G. S Chirikjian, "Diffusion-based motion planning for a nonholonomic flexible needle model," in Robotics and Automation, 2005. ICRA 2005. Proceedings of the 2005 IEEE International Conference on. IEEE, 2005, pp. 4600-4605.

[14] O. Khatib, "Real-time obstacle avoidance for manipulators and mobile robots," The international journal of robotics research, vol. 5, no. 1, pp. 90-98, 1986.

[15] P. Li, S. Jiang, J. Yang, and Z. Yang, "A combination method of artificial potential field and improved conjugate gradient for trajectory planning for needle insertion into soft tissue," Journal of Medical and Biological Engineering, vol. 34, no. 6, pp. 568-573, 2014.

[16] E. W. Dijkstra, "A note on two problems in connexion with graphs," Numerische mathematik, vol. 1, no. 1, pp. 269-271, 1959.

[17] S. M. LaValle, "Rapidly-exploring random trees: A new tool for path planning," 1998.

[18] M. Likhachev, D. I. Ferguson, G. J. Gordon, A. Stentz, and S. Thrun, "Anytime dynamic a*: An anytime, replanning algorithm." in ICAPS, 2005, pp. 262-271.

[19] F. Liu, A. Garriga-Casanovas, R. Secoli, and F. R. y Baena, "Fast and adaptive fractal tree-based path planning for programmable bevel tip steerable needles," IEEE Robotics and Automation Letters, vol. 1, no. 2, pp. 601-608, 2016.

[20] J. J. Kuffner and S. M. LaValle, "Rrt-connect: An efficient approach to single-query path planning," in Robotics and Automation, 2000. Proceedings. ICRA'OO. IEEE International Conference on, vol. 2. IEEE, 2000, pp. 995-1001.

[21] S. Karaman and E. Frazzoli, "Sampling-based algorithms for optimal motion planning," The international journal of robotics research, vol. 30, no. 7, pp. 846-894, 2011.

[22] M. Jordan and A. Perez, "Optimal bidirectional rapidly-exploring random trees," 2013.

[23] S. Patil and R. Alterovitz, "Interactive motion planning for steerable needles in 3d environments with obstacles," in Biomedical Robotics and Biomechatronics (BioRob), 2010 3rd IEEE RAS and EMBS International Conference on. IEEE, 2010, pp. 893-899.

[24] C. Caborni, S. Y. Ko, E. De Momi, G. Ferrigno, and F. R. y Baena, "Risk-based path planning for a steerable flexible probe for neurosurgical intervention," in Biomedical Robotics and Biomechatronics (BioRob), 2012 4th IEEE RAS \& EMBS International Conference on. IEEE, 2012, pp. 866-871.

[25] S. Y. Ko, L. Frasson, and F. R. y Baena, "Closed-loop planar motion control of a steerable probe with a programmable bevel inspired by nature," IEEE Transactions on Robotics, vol. 27, no. 5, pp. 970-983, 2011.

[26] J. D. Gammell, S. S. Srinivasa, and T. D. Barfoot, "Informed rrt*: Optimal sampling-based path planning focused via direct sampling of an admissible ellipsoidal heuristic," in Intelligent Robots and Systems
(IROS 2014), 2014 IEEE/RSJ International Conference on. IEEE, 2014, pp. 2997-3004.

[27] W. Sun, S. Patil, and R. Alterovitz, "High-frequency replanning under uncertainty using parallel sampling-based motion planning," IEEE Transactions on Robotics, vol. 31, no. 1, pp. 104-116, 2015. 